Does explaining the origins of misinformation improve the effectiveness of a given correction?

\author{
Saoirse Connor Desai \\ School of Psychology \\ University of New South Wales
}

Stian Reimers

Department of Psychology

City, University of London

Word count: 5868

\begin{abstract}
Author Note
Saoirse Connor Desai (iD https://orcid.org/0000-0001-7225-8973

Correspondence concerning this article should be addressed to Saoirse Connor

Desai, School of Psychology, University of New South Wales, 1006, Mathews Building,

8, Kensington NSW 2052; Email: s.connordesai@unsw.edu.au. Supplementary materials, data, and analyses are available at:https://osf.io/zjgx8/.
\end{abstract}




\begin{abstract}
Misinformation often has a continuing influence on event-related reasoning even when it is clearly and credibly corrected; this is referred to as the continued influence effect. The present work investigated whether a correction's effectiveness can be improved by explaining how the misinformation originated. In two experiments, we examined whether a correction that explained misinformation as originating from intentional deception, or an unintentional error were more effective than a correction that only identified the misinformation as false. Experiment 1 found that corrections which explained the misinformation as intentionally or unintentionally misleading were as effective as a correction that was not accompanied by an explanation for how the misinformation originated. We replicated this in Experiment 2 and found substantial attenuation of the continued influence effect in a novel scenario with the same underlying structure. Overall, the results suggest that informing people that the misinformation originated from a deliberate lie or accidental error may not be an effective correction strategy over and above stating that the misinformation is false. Keywords: Misinformation, Continued influence effect, Explanation, Correction
\end{abstract}




\section{Does explaining the origins of misinformation improve the effectiveness of a given correction?}

\section{Introduction}

People are often faced with information they subsequently learn is false. Incomplete, incorrect, and inaccurate reports often circulate through social media and journalistic channels, before eventually being corrected. Even when swiftly and credibly corrected, misinformation frequently has a continuing influence on memory and reasoning; this is known as the continued influence effect of misinformation (CIE; Chan et al., 2017; Ecker, Swire et al., 2014; Johnson \& Seifert, 1994; Lewandowsky et al., 2012; Sanderson et al., 2020; Walter \& Murphy, 2018; Walter \& Tukachinsky, 2020).

The growing abundance of misinformation in society, and the difficulties involved in correcting misinformation (Ecker, Lewandowsky, Swire et al., 2011), make establishing effective methods of correction all the more critical (Lewandowsky et al., 2017). The present study examines whether corrections that explain how the misinformation originated from a deliberate lie or an accidental error are more effective than corrections that only identify the information as false

\section{The continued influence effect}

The CIE is typically measured using a fictional scenario paradigm (see Johnson \& Seifert, 1994; Wilkes \& Leatherbarrow, 1988). In a standard CIE task, participants read a report of an unfolding event (e.g., a warehouse fire) that includes causal information (e.g., the fire was caused by carelessly stored flammable liquids), which is either presented and subsequently corrected (e.g., flammable liquids did not cause the fire), remains uncorrected, or is never presented at all (Bush et al., 1994; Connor Desai \& Reimers, 2019; Guillory \& Geraci, 2010; Hardwicke, 2016; Johnson \& Seifert, 1994; Wilkes \& Leatherbarrow, 1988). Participants' responses to later inference questions (e.g., "what could have caused the explosions?") often show that the corrected cause (i.e., there were flammable chemicals in the closet) has a continued influence on 
event-related reasoning, despite memory for event-related details remaining intact ${ }^{1}$.

Although a correction typically decreases reliance on misinformation relative to a no correction condition, it often fails to reduce reliance on misinformation to the level of a no misinformation baseline (Ecker, Lewandowsky \& Apai, 2011; Ecker, Lewandowsky, Swire et al., 2011; Ecker et al., 2010; Johnson \& Seifert, 1994), thereby eliminating its influence completely. The CIE has been demonstrated with different types of misinformation; for instance, with false beliefs about education (Ferrero, Hardwicke et al., 2020; Ferrero, Konstantinidis et al., 2020), political misinformation (Ecker \& Ang, 2019; Gordon et al., 2019; Guillory \& Geraci, 2016; Nyhan \& Reifler, 2015; Swire, Berinsky et al., 2017), commonly believed myths (e.g., liars give themselves away with physical tells; Swire, Ecker et al., 2017), as well as misinformation in newspaper headlines (Ecker, Lewandowsky, Chang et al., 2014).

The CIE has primarily been explained in terms of memory-updating and retrieval processes. The selective retrieval account holds that the CIE occurs when the misinformation is successfully retrieved but the correction is not (Ecker et al., 2017; Ecker, Lewandowsky \& Apai, 2011; Ecker, Lewandowsky, Swire et al., 2011; Ecker et al., 2010; Ecker, Swire et al., 2014; Lewandowsky et al., 2012). The model-updating account alternatively argues that the CIE is driven by a failure to integrate the updated information into a mental model of the described event constructed around the misinformation, unless an alternative explanation is available (Brydges et al., 2020; Gordon et al., 2017; Johnson \& Seifert, 1994; Kendeou et al., 2014; Wilkes \& Leatherbarrow, 1988). The selective retrieval account implies the misinformation can be retrieved without the correction whereas the model-updating account entails that although the specific information can be corrected the mental model is not.

\footnotetext{
${ }^{1}$ CIE studies often measure reliance on misinformation by coding responses to open-ended questions. However, the effect has also been observed with direct measures of continued reliance on misinformation, such as belief in the misinformation (Guillory \& Geraci, 2013; Rich \& Zaragoza, 2016), and closed-ended inference questions in which participants select options (Connor Desai \& Reimers,
} 2019) 


\section{Explaining misinformation}

Both selective retrieval and model-updating accounts tacitly assume that people's failure to disregard the misinformation is misguided. However, corrected misinformation may still have informational value, and it might be appropriate to treat it as, at least, partially informative if no grounds to disregard the misinformation have been provided (e.g., Kendeou et al., 2014; Swire, Berinsky et al., 2017). Corrected misinformation may, therefore, result in a CIE if people do not know why the misinformation was initially presented, or what led to it being corrected. In such cases, there may be no reason to place more weight on the correction than on the misinformation.

In the warehouse fire scenario described earlier, the correction (i.e., that flammable liquids initially thought to be in the warehouse were never actually there) does not explain why the misinformation was initially reported as true or why it is now considered to be false. Without explaining why the misinformation was reported, people might be unsure whether the correction is any more valid than the original misinformation, and consequently, prefer the first piece of information they encountered (Connor Desai et al., 2020).

Corrections that explain how the misinformation originated may address the conversational implications of the contradiction between misinformation; that is, the inferences people draw about what the speaker intends to communicate (Grice, 1975). On this view, corrections should be challenging when interpreting written or spoken statements, if the correction to misinformation only addresses the literal content of misinformation (e.g., there were no flammable chemicals on the premises), but not the conversational implications of misinformation (i.e., why was the misinformation reported in the first; Bush et al., 1994; Johnson \& Seifert, 1994; Seifert, 2002; Sperber et al., 2010).

Conversational conventions entail the assumption that speakers will not knowingly provide false information (Grice, 1975). Consequently, source characteristics, such as credibility, can affect the weight attached to a message or report (Hilton, 1990, 1995). Evidential reasoning studies have shown that reports from untrustworthy sources 
are treated with suspicion and potentially rejected (Pilditch et al., 2020), and that contradictions between testimonies can create distrust resulting in initial discounting of earlier witness reports (Connor Desai et al., 2016). Previous CIE studies have similarly shown that perceived source trustworthiness influences a correction's effectiveness such that corrections from low-trustworthiness sources are ineffective (Ecker \& Antonio, 2021; Guillory \& Geraci, 2013). Furthermore, the extent to which participants believe the correction is a predictor of its effectiveness (Ecker \& Antonio, 2021; Guillory \& Geraci, 2010; O'Rear \& Radvansky, 2020). The misinformation source's credibility is also argued to be strong determinant of the magnitude of CIE (Walter \& Tukachinsky, 2020). Corrections which inform people that the misinformation originated from a lie or an error could reduce the CIE by addressing the conversational implications of the correction, but may also make the misinformation's source appear less credible and therefore more likely to be discounted.

To date, there has been little research examining whether corrections that explain how the misinformation originated (i.e., from a lie or a mistake) are more effective than corrections that merely label the misinformation as false. As alluded to earlier, individuals confronted with correction to misinformation that does not explain how it originated may produce explanations that give the misinformation more weight if they are not provided with a compelling justification for why the original misinformation was conveyed (e.g., someone lied or made a mistake). For example, people might explain away the correction in the warehouse fire scenario by reasoning that the person who issued the correction was "paid off", or that a superior asked the employee to lie so as not to damage the companies' reputation. Even if corrected it might be reasonable to assume the truth is closer to the original (incorrect) statement than if it had not been issued (e.g., if it wasn't a flammable liquid then perhaps some other flammable substance; if the paint and gas cylinders were not stored in the closet perhaps they were stored elsewhere in the warehouse).

To the best of our knowledge, only one previous CIE study has directly examined the effectiveness of corrections that explain how the misinformation 
originated. Bush et al. (1994) examined whether corrections that explained the misinformation in terms of the communicative intentions of the misinformation were more effective than those that explain the literal content of the misinformation. They presented participants with the the warehouse fire scenario described earlier and examined the effectiveness of two explanatory corrections; the correction either explained why the misinformation may have been presented initially, but was irrelevant in the current context ("the expected delivery of paint and gas cylinders had not arrived"), or explained how the misinformation may have been presented in error ("the closet actually had coffee and soda cans rather than paint and gas cylinders). They found a marginal difference between the effectiveness of explanatory and non-explanatory corrections; however, explanatory corrections were insufficient to eliminate the CIE. However, Bush et al. (1994) did not directly examine the effectiveness of corrections which inform people that the misinformation was false because someone made a genuine mistake or intended to deceive.

In addition to examining whether corrections that explain the origins of the misinformation, the present work also sought to compare the relative effectiveness of corrections which explain that the misinformation was a genuine error against misinformation aimed to deliberately deceive. There are conflicting findings regarding whether informing people that a information source was intentionally deceptive or unintentionally erroneous facilitates discounting of original information. People discount eyewitness testimony when they are told that the eyewitness had a longstanding grudge against the suspect (Lagnado \& Harvey, 2008), and are more likely to discount an intentionally deceptive alibi than a mistaken alibi (Lagnado et al., 2013). Similarly, Fein et al. (1997) found that mock-jurors exposed to inadmissible evidence continued to be influenced by this information after instructions to disregard it, unless they were primed to be suspicious about the motives behind its introduction. These findings suggest that informing people that an information source was intentionally deceptive might facilitate discounting more that explaining that the misinformation originated from an error. Conversely, Green and Donahue (2011) found that participants did not correct their 
beliefs about a report when they were subsequently informed that the author of the report had "made it up" or that that report was inaccurate because of a "mix-up". The misinformation continued to influence participant's attitudes even when they were told the author had intentionally or unintentionally made up the content despite being motivated to correct for inaccuracies.

\section{Overview of Experiments}

Our chief aim was to examine whether corrections that explain the misinformation as intentionally or unintentionally misleading are more effective than corrections that do not explain how the misinformation originated. Providing an explanation for why misinformation is incorrect could facilitate correction processing (Brydges et al., 2020; Gordon et al., 2017; Johnson \& Seifert, 1994; Lewandowsky et al., 2012), or enhance later retrieval of the correction (Ecker, Lewandowsky, Swire et al., 2011; Gordon et al., 2017; Swire, Ecker et al., 2017). Alternatively, an explanation of deception or an oversight may be insufficient for individuals to reconsider the story and correct for inaccuracies (e.g., Green \& Donahue, 2011).

To investigate whether explaining how misinformation originated (intentionally or unintentionally) we presented participants with corrections which explained either that the misinformation was an accidental error or a deliberate lie. Experiment 1 tested the impact of explanatory corrections using the warehouse fire scenario. Experiment 2 then compared the effectiveness of explanatory and non-explanatory corrections in the warehouse fire scenario to a novel scenario describing a van crash. 


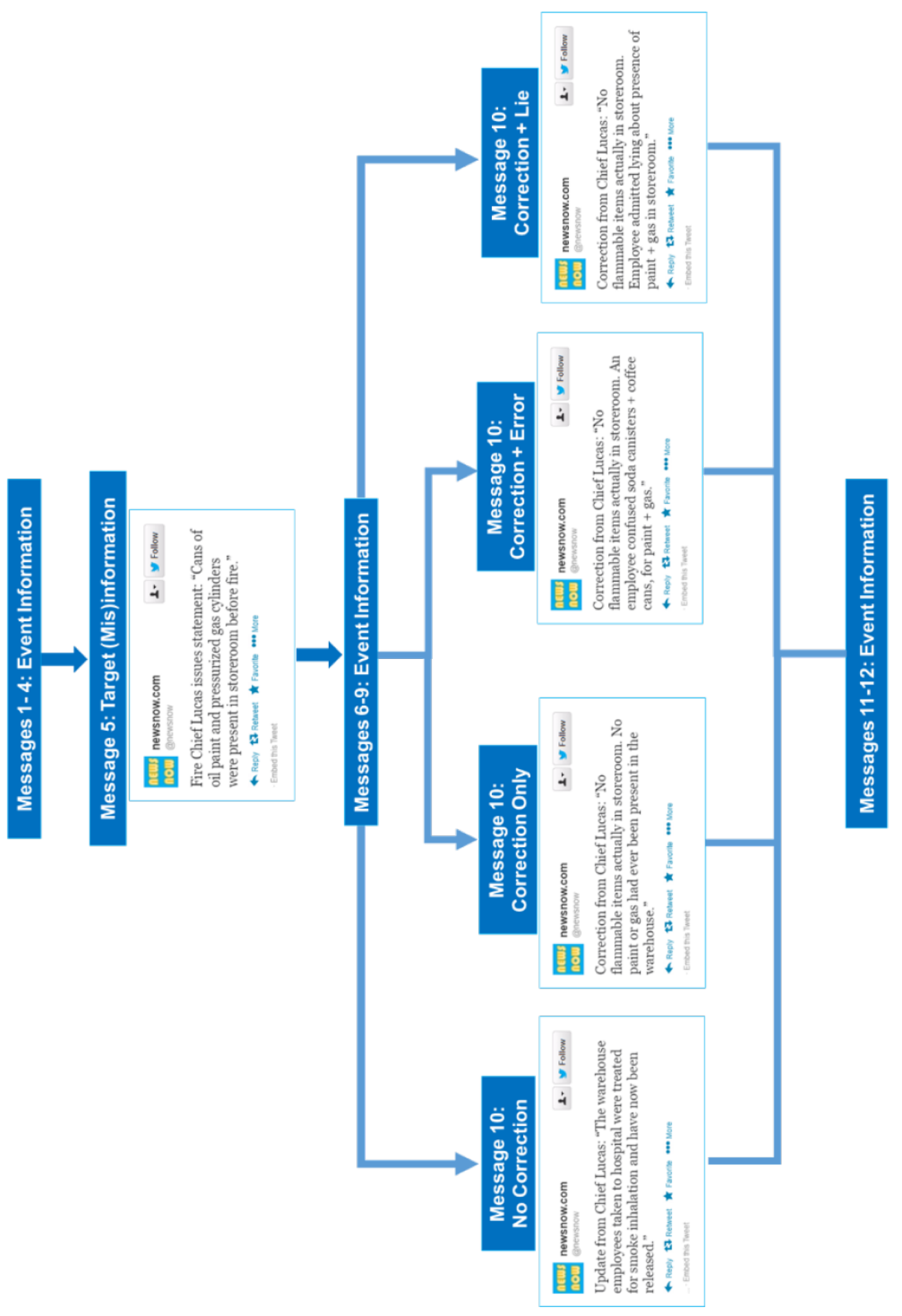

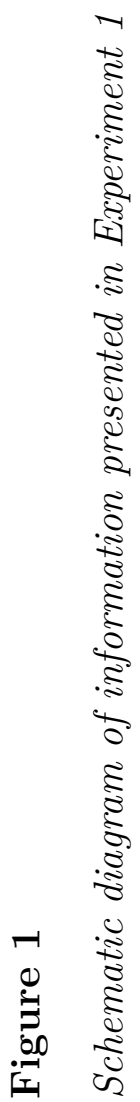




\section{Experiment 1}

Experiment 1 tested whether corrections, which explain that misinformation was announced either intentionally or unintentionally, are more effective than non-explanatory corrections. We tested this in the warehouse fire scenario described earlier but presented this as a series of social media posts. We predicted that a correction would reduce, but not eliminate, the number of references to misinformation compared to no correction, and that there would be fewer misinformation inferences following a correction that explains how the misinformation originated (i.e., lie or an error) than a correction that does not explain the misinformation's origins.

\section{Participants}

Three-hundred and sixty-five U.S. based participants were recruited via Amazon Mechanical Turk (MTurk) to retain at least 70 participants per condition. Only participants with a human intelligence task (HIT) approval rating greater than, or equal to $99 \%$, were recruited for the experiment. Participants had a mean age of 39.38 (SD = 11.92), and there were 169 females and 196 males. Participants were paid $\$ 1.50$ for their time $(M d n=16$ minutes $)$.

\section{Design and Materials}

We randomly assigned participants to one of four between-subjects conditions: the no correction, correction, correction + error explanation, or correction + lie explanation groups (see Figure 1). There were two primary measures: open-ended questions that required participants to make inferences about the scenario and questions on the scenario's details. We also asked the participants two questions aimed at assessing whether participants were aware of the correction. The primary dependent variable was the mean number of references to misinformation in response to the open-ended inference questionnaire.

Participants read one of four versions of a fictional news report about a fire at a stationery warehouse, each consisting of 12 sequentially-presented statements. The 
warehouse fire scenario has been used in several earlier continued influence effect studies (Connor Desai \& Reimers, 2019; Guillory \& Geraci, 2010; Johnson \& Seifert, 1994; Wilkes \& Leatherbarrow, 1988). We made two key changes the way the warehouse fire scenario in the present study. First, we modified the news report's presentation format, which we presented as a series of Tweets (cf. Hardwicke, 2016), to resemble the appearance of breaking news stories on Twitter. The Tweets originated from the same fictional news outlet, called "News Now" and did not exceed 140 characters (see Figure 1). Second, we amended the peripheral details that appeared either side of the misinformation and the correction, so they were neither consistent nor inconsistent with the causal explanation offered by the misinformation. The original warehouse fire story included statements that supported the misinformation that improperly stored chemicals caused the fire, such as "firefighters attending the scene report thick, oily smoke and sheets of flames hampering their efforts". In all other respects the scenario was the same use used in previous studies.

\section{Procedure}

Participants clicked on a link in MTurk to enter the experimental site. They subsequently read details about the experiment, gave consent, and completed an instructional manipulation check (IMC). The IMC required them to ignore the response format and confirm that they had read the instruction (Oppenheimer et al., 2009). Participants $(\mathrm{N}=4)$ who did not read the instructions accurately could not complete the study and received a message thanking them for their time.

The remaining participants received instructions that the study explored the factors that affect people's judgments about news reports. Their task was to read a brief report about an investigation into a fire, complete a short questionnaire about the report, and provide demographic information. We told participants that they would not be able to backtrack and that each message would appear for a minimum of 5 seconds before they could move on to the next message. Participants then read one of the four condition-dependent versions of the warehouse fire scenario. 
After reading the report, participants completed a questionnaire about the scenario: the questionnaire included seven inference questions, seven factual recall questions, and two questions probing awareness of the correction information. Inference and factual recall question blocks were intermixed and presented in random order, except the question probing the fire's most likely cause, which always came last. Participants typed a response to each of 16 questions in a text box, were required to use a minimum of 25 characters, and encouraged to answer using full sentences. After completing the questionnaire, participants provided their sex, age, and their highest level of education.

\section{Results}

The analysis for this and all subsequent experiments was carried out with JASP v0.10.2 using the default prior scale (Rouder et al., 2017). We followed Lee and Wagenmakers (2014 suggested conventions that a $\mathrm{BF}_{10}$ between 3 and 10 (0.33-0.1) represents "moderate" evidence for the alternative (or null) hypotheses, a $\mathrm{BF}_{10}$ between 10-30 (0.1-0.03) represents "strong" evidence", a $\mathrm{BF}_{10}$ between 30-100 (0.03-0.01) represents "very strong evidence", and a $\mathrm{BF}_{10}$ above $100(<0.01)$ represents "extreme evidence". ${ }^{2}$.

\section{Coding of Responses}

We used responses to three types of questions in the analysis. Participants answered the seven inference questions based on their understanding of the report. Responses to inference questions were coded as a reference to misinformation if they explicitly stated or strongly implied that oil paint and gas cylinders caused or contributed to the fire and were scored 0 otherwise. The minimum inference score was 0 and the maximum of 7 . Participants could answer the factual recall questions by recalling the details of the report. Each response was coded as one when the participant

\footnotetext{
${ }^{2}$ We also performed frequentist analyses of the data. Details of both the Bayesian and frequentist analyses can be found at https://osf.io/zjgx8/.
} 
fully or partially recalled the detail and scored 0 if it was not. There was a minimum recall accuracy score of 0 and a maximum score of 7 . We computed awareness of correction scores using the same criteria; the maximum individual critical information score was two.

\section{Inter-coder reliability}

All responses were coded by a scorer who was naive to the experimental conditions using a standardized scoring guide. A second, independent coder received instructions on the coding scheme and coded $10 \%$ of participants' responses $(\mathrm{n}=36)$. Inter-rater agreement was 0.88 and Cohen's $\kappa=0.76 \pm 0.03$, indicating a high level of agreement between coders, both of which are higher than the benchmark values of 0.7 and 0.6 (Landis \& Koch, 1977).

\section{Inference Scores}

Figure 2 shows that explanatory and non-explanatory corrections types reduced but did not eliminate continued references to misinformation. There was very strong evidence for the effect of correction $\mathrm{BF}_{10}>10,000$. Post-hoc comparisons provided very strong evidence for the difference that the explanatory and non-explanatory correction conditions differed from the no correction condition, $\mathrm{BF}_{10}$ 's $>10,000$. However, there was moderate evidence against differences between the correction conditions, $\mathrm{BF}_{10}$ 's $<$ 0.206.

\section{Correction acknowledgment}

A key claim from the CIE literature is that people often continue to rely on misinformation despite clearly understanding and recalling that the misinformation was corrected (Johnson \& Seifert, 1994). We tested this by calculating the proportion of participants who correctly recalled the correction and referred to misinformation in response to at least one inference question. A substantial minority of participants in each correction condition made at least one reference to misinformation on inference questions while acknowledging the correction: $27 \%$ of the correction only group, $23 \%$ of 




\section{Figure 2}

Correction Information

Violin plots show the distribution and probability density of references to misinformation by correction information condition in Experiment 1. The violin plot is a symmetrical rotated kernel density plot and shows the density of the data at different values. Black points represent mean and 95\% confidence interval of the mean. Dashed lines represent condition means after excluding participants who did not recall the correction. Colored dots show individual data points.

the correction + error group, and $28 \%$ of the correction + lie group. A small majority of participants (36-44\%) showed no continued influence effect; that is, they accurately recalled the correction and made no explicit references to misinformation. The remaining participants (13-20\%) did not recall the correction information. Overall, we observed that a substantial proportion of participants who received a correction continued to refer to misinformation despite acknowledging the correction.

\section{Recall Accuracy Scores}

Participants in the no correction $(M=4.43, S D=1.85)$, correction only $(\mathrm{M}=$ 4.36, $S D=1.70)$, correction + error $(M=4.76, S D=1.65)$, and correction + lie $(M=$ $4.30, S D=1.86)$ groups performed equally well on recall accuracy measures. There was 
no evidence for the effect correction information on recall accuracy, $\mathrm{BF}_{10}=0.049$, suggesting that differences in factual recall of story information cannot account for the differences in inference scores between conditions.

\section{Discussion}

This experiment replicated the continued influence effect of misinformation; a correction reduced but did not eliminate references to misinformation. There was moderate evidence that corrections which explained how misinformation originated did not differ from corrections did not differ corrections that did not explain the misinformation's origins. Explanatory corrections were, therefore, as effective as non-explanatory corrections and reduced references to corrected misinformation equally. Almost a third of participants in each of the three correction groups made at least one reference to misinformation and exhibited a CIE. The present results strengthen previous findings that addressing the conversational implications of the misinformation reduces but does not eliminate the continued influence effect (Seifert, 2002). Moreover, informing people that the original misinformation source was intentionally deceptive or made an accidental error was (Green \& Donahue, 2011) does not result in people correcting for inaccuracies effectively. These results suggest that there is no additional benefit gained by explaining that a misinformation's originated from a lie or an error.

To corroborate this finding, in Experiment 2, we examined the effectiveness of corrections that explain how the misinformation originated in a novel scenario. Establishing whether or not an experimental effect is present with a single stimulus scenario can limit the scope of the conclusions reached. Including multiple scenario versions can therefore increase confidence that the results generalize across scenarios (Monin \& Oppenheimer, 2014; Westfall et al., 2015). Accordingly, Experiment 2 compared inferences from the warehouse fire scenario to a scenario with the same underlying structure, but a different subject-matter, to examine whether the null effect of explanatory corrections extended to other scenarios. 




\section{Experiment 2}

Experiment 2 explored the effect of explanatory and non-explanatory corrections to misinformation in the warehouse fire scenario and a new scenario describing a van crash. The scenarios in Experiment 2 also included a statement describing other potential causes of the outcome described in the scenario (e.g., common causes of industrial fires). The potential cause statement appeared immediately before the misinformation so that it was possible to make causal inferences without referring to the misinformation and increase the availability of alternative causes in memory.

\section{Participants}

One-hundred and sixty-three participants completed the experiment via MTurk with the intention to retain 20 participants per condition. Four participants were excluded before analysis because they failed a recognition test of the correction/control message. Another participant wrote nonsensical responses, so their responses were excluded from the analysis. We included one-hundred and fifty-eight participants in the final analysis. Participants had a mean age of $39.62(S D=11.21)$, and there were 69 females and 89 males. Participants were paid $\$ 1.50$ for their time ( $M d n=16$ minutes).

\section{Design and Materials}

A 2 (Scenario: Van Crash, Warehouse Fire) x 4 (Correction Information: No Correction, Correction Only, Correction + Error, Correction + Lie) between-subjects factorial design was used such that there were four versions of the warehouse fire and the van crash scenario (see Appendix B for an overview of the scenario structure). There was a random allocation of participants to one of the eight experimental conditions.

The materials consisted of two different scenarios (warehouse fire, van crash) presented in individual breaking news statements originating from the same fictional news source. The maximum character length per message increased from 140 to 280 to allow additional information in the explanatory correction messages. Messages were approximately matched for the number of characters and words across experimental 
conditions.

The new scenario described a van that had crashed while returning from a festival. The misinformation suggested that the van had crashed because the driver had been drinking (see Appendix B). To directly compare between scenarios, we modeled the inference questions for the van crash scenario on those used for the warehouse fire scenario in order. For example, an inference question for the warehouse fire scenario asked, "How could the fire at the warehouse have been avoided?" and for the van crash scenario, similarly asked, "How could this accident have been avoided?".

\section{Procedure}

All elements of the experimental procedure were identical to those of Experiment 1, except as stated below. We changed the instruction check and added a recognition test examining whether participants had encoded the correction. The instruction check appeared after reading instructions and before beginning the experiment. Participants answered three questions about the main instructions (e.g., what is the minimum time each statement will appear on the screen?). We included this instruction check to encourage participants' attentiveness throughout the experiment, but there was no consequence of failing the test. The instruction check was updated to be consistent with developing best practice in the field (Berinsky et al., 2014, 2016; Hauser \& Schwarz, 2016).

\section{Results}

\section{Coding of Responses}

Responses that explicitly stated or strongly implied that the target misinformation was causally involved in the event were scored 1 and otherwise scored 0 (e.g., "the van crashed because the driver was drunk"). For the warehouse fire scenario, we did not count references to flammable materials that did not explicitly mention storage (e.g., "It could have been avoided by keeping flammable objects or items in place"). Similarly, in the van crash scenario, references to driver behavior that did not 
mention intoxication or drunkenness concerning the van crashing were not counted as references to misinformation (e.g., "by having him be more alert drinking coffee"). The maximum individual inference score was 7 . We applied the same coding criteria from Experiment 1 to fact recall and critical information recall responses.

\section{Inter-coder reliability}

A coder who was naive to experimental conditions scored all responses. A second, independent judge then coded approximately $10 \%$ of participants' responses. Inter-rater agreement was 0.90 and Cohen's $\kappa=0.81 \pm 0.05$, indicating a very high level of agreement between coders.

\section{Inference Scores}

Figure 4 shows that both explanatory and non-explanatory corrections were equally effective in reducing references to misinformation, but that corrections were generally more effective in the van crash scenario. A Bayesian ANOVA was used to determine the effects of correction information and scenario on inference scores, and revealed very strong evidence for the main effect of correction information, $\mathrm{BF}_{10}>$ 10,000 , strong evidence for the main effect of scenario, $\mathrm{BF}_{10}=29.25$, and the interaction between correction information and scenario, $\mathrm{BF}_{10}=117.62$. As shown in Figure 4, participants who received no correction made more references to misinformation for the van crash than the warehouse fire scenario. In contrast, participants who received a correction made fewer references to misinformation for the van crash than the warehouse fire scenario. ${ }^{3}$

\footnotetext{
${ }^{3}$ In a pre-registered follow-up study we checked whether greater attenuation of the CIE in the van crash than the warehouse fire scenario was due to the way the correction invalidated the misinformation in the former. The method, directional hypotheses, and analysis plan (including planned analyses, data stopping rule, and exclusion criteria) before data collection; can be found at https://osf.io/ywj8d/. Including and removing 'evidence' from the corrections did not change the difference between scenarios and the number of references to misinformation following a correction were substantially attenuated in the van crash scenario when compared to the warehouse fire scenario. We performed Bayesian analyses consistent with the experiments reported here. Details of the analyses can
} 


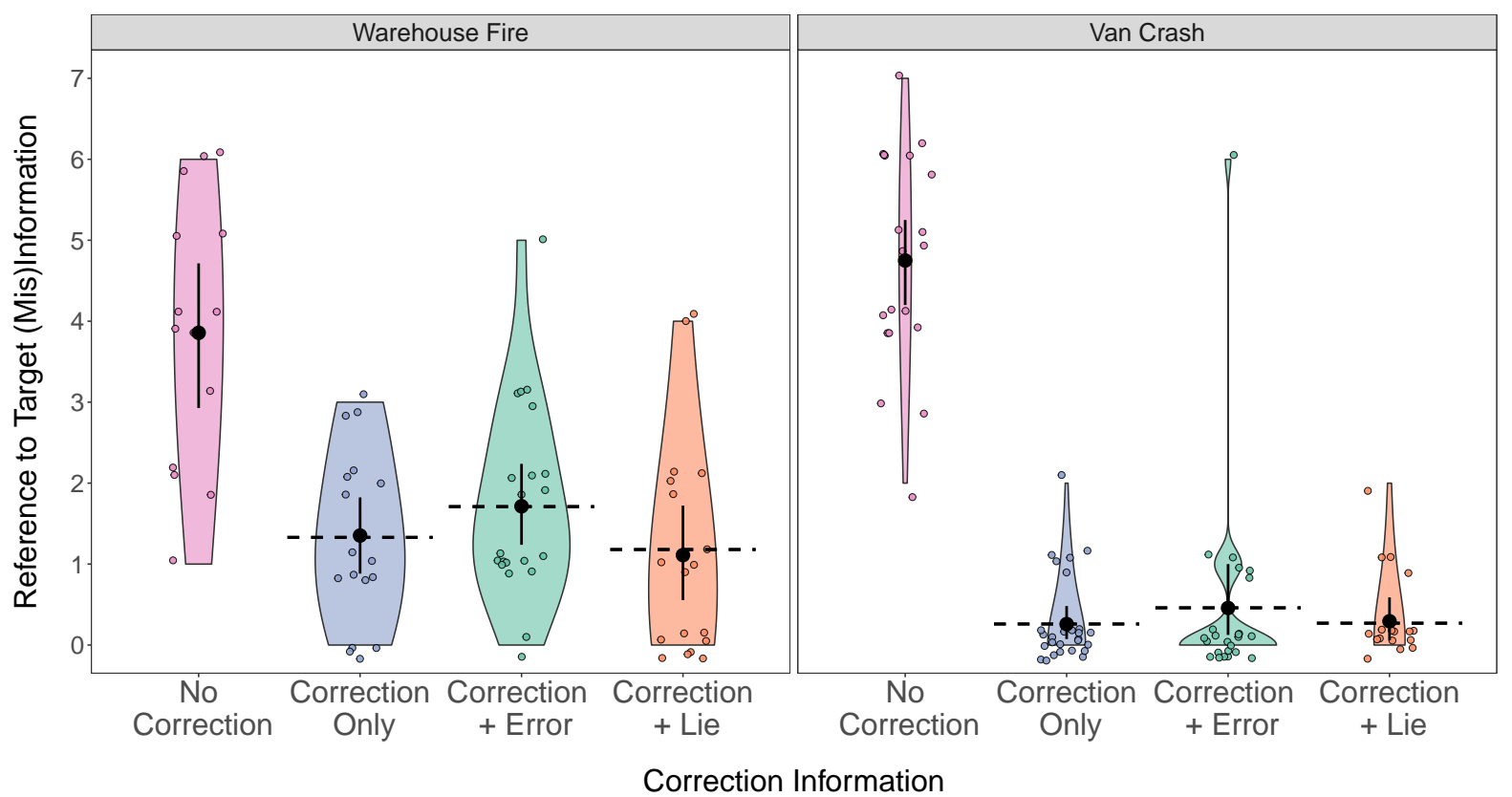

\section{Figure 4}

Violin plots show the distribution and probability density of references to misinformation by correction information condition and scenario in Experiment 2. Black points represent mean and $95 \%$ confidence interval of the mean. Dashed lines represent condition means after excluding participants who did not recall the correction. Colored dots show individual data points.

Although the effect of correction appeared to be more robust for the van crash scenario, looking at each scenario separately, it was clear the effect of all types of correction was also substantial for the warehouse scenario. Post-hoc comparisons for the warehouse fire scenario further revealed very strong evidence that explanatory and non-explanatory corrections reduced reliance on misinformation compared to the no correction condition, $\mathrm{BF}_{10}$ 's $>200$. The evidence for the difference between correction conditions moderately favored the null, $\mathrm{BF}_{10}$ 's $<0.8$. Post hoc comparisons for the van crash scenario similarly revealed very strong evidence that explanatory and non-explanatory corrections reduced misinformation references compared to the no correction condition, $\mathrm{BF}_{10}$ 's $>10,000$. The evidence for the difference between the three correction conditions was in favour of the null, $\mathrm{BF}_{10}$ 's $<0.36$.

be found at https://osf.io/zjgx8/. 


\section{Correction acknowledgement}

In the warehouse fire scenario, $81 \%$ of participants in the correction + error group referred to misinformation at least once and acknowledged the correction; this was $50 \%$ in the correction + lie group, and $65 \%$ in the correction only group. Between 5-33\% of participants who read the warehouse fire scenario showed no continued influence effect; they accurately recalled the correction and made no references to the misinformation. The remaining $5-12 \%$ of participants did not recall the correction when probed.

In contrast, in the van crash scenario, $25 \%$ of participants in the correction + error group; $18 \%$ of the correction + lie condition; and $22 \%$ of the correction only group referred to the misinformation at least once whilst also acknowledging that it had been corrected. The majority of participants (65-77\%) showed no continued influence effect and accurately recalled the correction. Between 0-12\% of participants who read the van crash scenario did not recall the correction.

Notably, more the twice the number of participants referred to misinformation and acknowledged the correction after reading the warehouse fire scenario than the van crash scenario.

\section{Recall Accuracy Scores}

There was substantial evidence for the main effect of scenario, $\mathrm{BF}_{10}=5.219$ on recall accuracy. Participants recalled more details from the van crash scenario $(\mathbf{M}=$ $5.56, \mathrm{SD}=1.35)$ than the warehouse fire scenario $(\mathrm{M}=4.83, \mathrm{SD}=1.83)$. The evidence for the main effect of correction information and interaction between correction information and scenario, $\mathrm{BF}_{10}$ 's $<0.17$, were in favor of the null. However, the main effect of scenario was not particularly noteworthy given that the scenarios had different questions. The evidence for the null effect of correction conditions within scenarios was key to establishing whether participants in some correction conditions recalled more than others.

Although the two scenarios necessarily required different questions to test 
memory for the scenario details, the difference in recall scores potentially suggested that people found the van crash scenario easier to represent. Therefore, one potential explanation for the more substantial attenuation of the continued influence effect in the van crash scenario compared to the warehouse fire scenario, could be the difference in recall performance between scenarios. As participants recalled more details from the van crash than the warehouse fire scenario, better memory for the scenario could explain why the correction was more effective in the former scenario than the latter.

\section{Median-split analysis}

To examine whether differences in the effectiveness of the corrections between scenarios reflected better memory for scenario details, we looked within scenarios to examine whether participants who more accurately recalled scenario details showed less of a CIE. We first excluded responses to the recall accuracy question that referred to the misinformation (i.e., What was present in the warehouse before the fire? vs What was the driver drinking at the service station?), then divided participants into higher and lower recall groups based on median splits on their recall accuracy scores, and examined the evidence for a difference in inference scores between the two groups. The analysis revealed moderate evidence for the null effect of accuracy group (high vs low), $\mathrm{BF}_{10}=0.234$, and scenario, $\mathrm{BF}_{10}=0.578$, and interaction between accuracy group and scenario, $\mathrm{BF}_{10}=0.145$ on inference scores; thus indicating that the difference in the magnitude of the continued influence effect between scenarios was not due to better memory for scenario details.

\section{Discussion}

Experiment 2 replicated Experiment 1's findings showing that corrections which explain how the misinformation originated were as effective as corrections that did not explain how the misinformation transpired. Both explanatory and non-explanatory corrections were equally effective at reducing the CIE. Explanatory corrections in both the warehouse fire and van crash scenarios were no more effective as non-explanatory corrections. Both explanatory and non-explanatory corrections reduced, but did not 
eliminate, the CIE for the warehouse fire scenario. Corrections in the van crash scenario were markedly more effective than in the warehouse fire scenario, however, irrespective of whether the correction explained the misinformation or not. Experiment 2's results provide further confirmation that corrections that explain how the misinformation originated (either from deliberate deceit or an accidental error) are as effective as corrections that label the misinformation as false.

\section{General Discussion}

The present study examined whether explaining that misinformation originated from an intentional deception or from an unintentional error can improve a correction's effectiveness relative to a correction that merely labels the misinformation as false. In two experiments, we found evidence that corrections which explained the misinformation as originating from a lie or an error were as effective as a correction which identified the misinformation as false. Corrections that explained misinformation as intentionally deceptive (i.e., a lie) were also as effective as corrections that explained misinformation as an unintentional error (Green \& Donahue, 2011).

These results suggest that informing people that misinformation originated from a lie or an error may not reduce the sufficiently reduce the CIE over corrections which only state that the information is false. Our findings are consistent with previous studies showing that corrections which accounted for the conversational implications of the misinformation (e.g., "X, which had originally been believed because of $\mathrm{Y}$, is actually untrue") somehwat reduce, but do not eliminate the CIE (Bush et al., 1994). The results are also consistent with work showing that people continue to believe stories after learning that the story contained inaccuracies due to an error or author deception (Green \& Donahue, 2011). Consistent with previous studies on the CIE, all three types of correction reduced reliance on misinformation relative to the no correction control condition (e.g., Ecker et al., 2017; Ecker, Lewandowsky, Swire et al., 2011), but did not eliminate the CIE entirely.

The current study provides evidence that people continue to rely on corrected 
misinformation even when they know it originated from intentional deception or an unintentional error. Participant's event-related reasoning was influenced by misinformation despite being told that the misinformation originated from a lie or a mistake. Both the model-updating (Brydges et al., 2020; Gordon et al., 2017; Johnson \& Seifert, 1994; O’Rear \& Radvansky, 2020; Rich \& Zaragoza, 2016), and selective retrieval (Ecker, Lewandowsky, Swire et al., 2011; Gordon et al., 2017; Swire, Ecker et al., 2017) accounts, suggest that more detailed corrections which provide an explanation for why the misinformation is incorrect are more effective (Swire, Ecker et al., 2017). On this view, explaining that the misinformation was initially conveyed in error or to deceive should increase the correction's salience and encourage more elaborate processing, making it more likely to be integrated during encoding, or successfully retrieved later. Our results do not appear to fit with either a model-updating or selective retrieval account of the CIE. Informing people that misinformation was intentionally deceptive or an accidental error does not appear to facilitate updating or boost retrieval of the correction in the way that detailed explanations for why the misinformation is incorrect do (Kendeou et al., 2014; Swire, Ecker et al., 2017).

As mentioned earlier, the finding that corrections explaining the origins of the misinformation are as effective as corrections identifying the information as false is consistent with previous studies (Bush et al., 1994; Green \& Donahue, 2011). Our results also fit with previous studies on evidential reasoning showing that people make erroneous inferences when faced with a deceptive source (Pilditch et al., 2019). These results are, however, inconsistent with previous work showing that perceived source credibility moderates the effectiveness of corrective information (Ecker \& Antonio, 2021; Guillory \& Geraci, 2013), suggesting explaining that the misinformation originated from a lie or an error did not impact the credibility of the person that issued the misinformation statement. An interesting extension of this work would be to examine how directly discrediting the person issuing the correction affects continued reliance on misinformation.

There are several potential explanations for the relative ineffectiveness of 
corrections that explain how the misinformation originated in the current study. As mentioned previously, corrections which provide more detailed explanations for an inaccuracy are more effective than less detailed explanations (e.g., Ecker et al., 2020; Swire, Berinsky et al., 2017; Swire, Ecker et al., 2017). One interpretation of the present results is that the explanation offered for how the misinformation originated was not detailed enough or did not sufficiently explain its origins; and consequently, did not reduce the CIE further than a correction with no explanation. Examining how the level of detail provided in an explanation for the misinformation's origin would establish the extent to which detailed explanation about the misinformation's origin impacts a correction's effectiveness.

Causal explanations can also be viewed as a form of social interaction and therefore subject to rules of conversation (Hilton, 1990). The social nature of the explanations provided in the present work could have constrained the effectiveness of the correction such that any pragmatic benefit gained from the explanation of the misinformation's invalidity was nullified because it created a new set of inferences about why an employee lied, or made an error. A more direct way to test the effect of such explanatory corrections would be to explain why the person issuing the statement is unreliable rather than an unseen misinformation source who was not previously mentioned. Relatedly, the fact that the person who initially conveyed the misinformation failed to provide true or relevant information could also result in people disregarding the explanation and focusing on the negation (i.e., that there were no flammable substances in the storeroom). Some courtroom simulation studies suggest that people are more convinced by physical evidence than eyewitness evidence (e.g., Skolnick \& Shaw, 2001), perhaps because people assume that human measuring devices (e.g. eyewitness) are inherently less reliable than physical ones (e.g. CCTV; see Lagnado et al., 2013. Explanatory corrections may be more effective if the correction involved a physical explanation of why misinformation is incorrect rather than a social explanation of a misunderstanding or deception. For example, if the correction had stated that oil paint and gas cylinders had been moved to a fire-secure room in a 
different building and therefore could not have caused the fire.

Instilling a sense of distrust about the misinformation source's motives may also be more effective at encoding than at retrieval (i.e., when misinformation is encoded rather than when the correction is presented). This explanation also fits with recent work showing that people are better able to incorporate information about constraints on a sample of evidence when it presented at encoding than when presented at retrieval (Ransom et al., 2021).

Our primary goal was to examine whether the continued influence effect of misinformation could be more effectively reduced, or eliminated, when corrected by informing participants that it stemmed from a lie or an error, than when no explanation for its origins is given. In Experiment 1 we found explanatory and non-explanatory corrections to be equally effective at reducing the CIE in the warehouse fire scenario. In Experiment 2 we replicated this finding and examined whether it generalized to a new scenario involving a van crash. Results showed the CIE was substantially attenuated in the van crash scenario compared to the warehouse fire scenario. Although inter-scenario differences in the magnitude of the CIE was not the primary focus of our investigation, the discrepancy was unexpected. Future studies could examine the types of causal scenarios that give rise to the CIE to establish the boundary conditions of the effect.

\section{Conclusions}

The current studies provide clear evidence that explaining the reason for the presentation of misinformation, whether the cause was an understandable mistake or dishonesty, does not necessarily reduce people's reliance on misinformation in generating causal explanations for an event. These findings have crucial real-world implications and suggest that people may continue to believe misinformation even after learning that it was mistaken or deceitful. The present studies re-confirmed that corrections often reduce the continuing influence of misinformation but do not eliminate it completely. The present work advances understanding of the cognitive mechanisms involved the drive the continued influence effect of misinformation and point to 
promising avenues for future research. 


\section{References}

Berinsky, A. J., Margolis, M. F. \& Sances, M. W. (2014). Separating the shirkers from the workers? making sure respondents pay attention on self-administered surveys. American Journal of Political Science, 58(3), 739-753.

Berinsky, A. J., Margolis, M. F. \& Sances, M. W. (2016). Can we turn shirkers into workers? Journal of Experimental Social Psychology, 66, 20-28.

Brydges, C. R., Gordon, A. \& Ecker, U. K. (2020). Electrophysiological correlates of the continued influence effect of misinformation: An exploratory study. Journal of Cognitive Psychology, 32(8), 771-784.

Bush, J., Johnson, H. \& Seifert, C. M. (1994). The implications of corrections: Then why did you mention it? Proceedings of the sixteenth annual conference of the cognitive science society, 112-117.

Chan, M.-p. S., Jones, C. R., Hall Jamieson, K. \& Albarracin, D. (2017). Debunking: A meta-analysis of the psychological efficacy of messages countering misinformation. Psychological science, 28(11), 1531-1546.

Connor Desai, S., Reimers, S. \& Lagnado, D. (2016). Consistency and credibility in legal reasoning: A bayesian network approach. Proceedings of the 38th Annual Conference of the Cognitive Science Society, 626-631.

Connor Desai, S., Pilditch, T. D. \& Madsen, J. K. (2020). The rational continued influence of misinformation. Cognition, 205, 104453.

Connor Desai, S. \& Reimers, S. (2019). Comparing the use of open and closed questions for web-based measures of the continued-influence effect. Behavior research methods, $51(3), 1426-1440$.

Ecker, U. K. \& Ang, L. C. (2019). Political attitudes and the processing of misinformation corrections. Political Psychology, 40(2), 241-260.

Ecker, U. K. \& Antonio, L. M. (2021). Can you believe it? an investigation into the impact of retraction source credibility on the continued influence effect. Memory E Cognition, $49(4), 631-644$. 
Ecker, U. K., Hogan, J. L. \& Lewandowsky, S. (2017). Reminders and repetition of misinformation: Helping or hindering its retraction? Journal of Applied Research in Memory and Cognition, 6(2), 185-192.

Ecker, U. K., Lewandowsky, S. \& Apai, J. (2011). Terrorists brought down the plane! - no, actually it was a technical fault: Processing corrections of emotive information. Quarterly Journal of Experimental Psychology, 64(2), 283-310.

Ecker, U. K., Lewandowsky, S., Chang, E. P. \& Pillai, R. (2014). The effects of subtle misinformation in news headlines. Journal of experimental psychology: applied, $20(4), 323$.

Ecker, U. K., Lewandowsky, S., Swire, B. \& Chang, D. (2011). Correcting false information in memory: Manipulating the strength of misinformation encoding and its retraction. Psychonomic Bulletin \& Review, 18(3), 570-578.

Ecker, U. K., Lewandowsky, S. \& Tang, D. T. (2010). Explicit warnings reduce but do not eliminate the continued influence of misinformation. Memory $\&$ cognition, $38(8), 1087-1100$.

Ecker, U. K., O'Reilly, Z., Reid, J. S. \& Chang, E. P. (2020). The effectiveness of short-format refutational fact-checks. British Journal of Psychology, 111(1), $36-54$.

Ecker, U. K., Swire, B. \& Lewandowsky, S. (2014). Correcting misinformation-a challenge for education and cognitive science.

Fein, S., McCloskey, A. \& Tomlinson, T. (1997). Can the jury disregard that information? the use of suspicion to reduce the prejudicial effects of pretrial publicity and inadmissible testimony. Personality and Social Psychology Bulletin, 23(11), 1215-1226.

Ferrero, M., Hardwicke, T. E., Konstantinidis, E. \& Vadillo, M. A. (2020). The effectiveness of refutation texts to correct misconceptions among educators. Journal of experimental psychology: applied. 
Ferrero, M., Konstantinidis, E. \& Vadillo, M. A. (2020). An attempt to correct erroneous ideas among teacher education students: The effectiveness of refutation texts. Frontiers in psychology, 11, 2704.

Gordon, A., Brooks, J. C., Quadflieg, S., Ecker, U. K. \& Lewandowsky, S. (2017). Exploring the neural substrates of misinformation processing. Neuropsychologia, $106,216-224$.

Gordon, A., Ecker, U. K. \& Lewandowsky, S. (2019). Polarity and attitude effects in the continued-influence paradigm. Journal of Memory and Language, 108, 104028.

Green, M. C. \& Donahue, J. K. (2011). Persistence of belief change in the face of deception: The effect of factual stories revealed to be false. Media Psychology, $14(3), 312-331$.

Grice, H. P. (1975). Logic and conversation. Speech acts (pp. 41-58). Brill.

Guillory, J. J. \& Geraci, L. (2010). The persistence of inferences in memory for younger and older adults: Remembering facts and believing inferences. Psychonomic Bulletin 8 Review, 17(1), 73-81.

Guillory, J. J. \& Geraci, L. (2013). Correcting erroneous inferences in memory: The role of source credibility. Journal of Applied Research in Memory and Cognition, 2(4), 201-209.

Guillory, J. J. \& Geraci, L. (2016). The persistence of erroneous information in memory: The effect of valence on the acceptance of corrected information. Applied Cognitive Psychology, 30(2), 282-288.

Hardwicke, T. E. (2016). Persistence and plasticity in the human memory system: An empirical investigation of the overwriting hypothesis (Doctoral dissertation). UCL (University College London).

Hauser, D. J. \& Schwarz, N. (2016). Attentive turkers: Mturk participants perform better on online attention checks than do subject pool participants. Behavior research methods, 48(1), 400-407.

Hilton, D. J. (1990). Conversational processes and causal explanation. Psychological Bulletin, $107(1), 65$. 
Hilton, D. J. (1995). The social context of reasoning: Conversational inference and rational judgment. Psychological bulletin, 118(2), 248.

Johnson, H. M. \& Seifert, C. M. (1994). Sources of the continued influence effect: When misinformation in memory affects later inferences. Journal of Experimental Psychology: Learning, Memory, and Cognition, 20(6), 1420.

Kendeou, P., Walsh, E. K., Smith, E. R. \& O’Brien, E. J. (2014). Knowledge revision processes in refutation texts. Discourse Processes, 51 (5-6), 374-397.

Lagnado, D., Fenton, N. \& Neil, M. (2013). Legal idioms: A framework for evidential reasoning. Argument \& Computation, 4(1), 46-63.

Lagnado, D. \& Harvey, N. (2008). The impact of discredited evidence. Psychonomic bulletin \& review, 15(6), 1166-1173.

Landis, J. R. \& Koch, G. G. (1977). The measurement of observer agreement for categorical data. biometrics, 159-174.

Lee, M. D. \& Wagenmakers, E.-J. (2014). Bayesian cognitive modeling: A practical course. Cambridge university press.

Lewandowsky, S., Ecker, U. K. \& Cook, J. (2017). Beyond misinformation:

Understanding and coping with the "post-truth" era. Journal of applied research in memory and cognition, 6(4), 353-369.

Lewandowsky, S., Ecker, U. K., Seifert, C. M., Schwarz, N. \& Cook, J. (2012). Misinformation and its correction: Continued influence and successful debiasing. Psychological science in the public interest, 13(3), 106-131.

Monin, B. \& Oppenheimer, D. M. (2014). The limits of direct replications and the virtues of stimulus sampling.

Nyhan, B. \& Reifler, J. (2015). Displacing misinformation about events: An experimental test of causal corrections.

Oppenheimer, D. M., Meyvis, T. \& Davidenko, N. (2009). Instructional manipulation checks: Detecting satisficing to increase statistical power. Journal of experimental social psychology, 45(4), 867-872. 
O'Rear, A. A. \& Radvansky, G. A. (2020). Failure to accept retractions: A contribution to the continued influence effect. Memory \& cognition, 48(1), 127-144.

Pilditch, T. D., Fries, A. \& Lagnado, D. A. (2019). Deception in evidential reasoning: Willful deceit or honest mistake? CogSci, 931-937.

Pilditch, T. D., Madsen, J. K. \& Custers, R. (2020). False prophets and cassandra's curse: The role of credibility in belief updating. Acta psychologica, 202, 102956.

Ransom, K., Perfors, A., Hayes, B. \& Connor Desai, S. (2021). What do our sampling assumptions affect: How we encode data or how we reason from it? https://doi.org/10.31234/osf.io/yqtd9

Rich, P. R. \& Zaragoza, M. S. (2016). The continued influence of implied and explicitly stated misinformation in news reports. Journal of experimental psychology: learning, memory, and cognition, 42(1), 62.

Rouder, J. N., Morey, R. D., Verhagen, J., Swagman, A. R. \& Wagenmakers, E.-J. (2017). Bayesian analysis of factorial designs. Psychological Methods, 22(2), 304.

Sanderson, J. A., Ecker, U. K. \& Sanderson, J. (2020). The challenge of misinformation and ways to reduce its impact. Handbook of Learning from Multiple Representations and Perspectives.

Seifert, C. M. (2002). The continued influence of misinformation in memory: What makes a correction effective? Psychology of learning and motivation (pp. 265-292). Elsevier.

Skolnick, P. \& Shaw, J. I. (2001). A comparison of eyewitness and physical evidence on mock-juror decision making. Criminal Justice and Behavior, 28(5), 614-630.

Sperber, D., Clément, F., Heintz, C., Mascaro, O., Mercier, H., Origgi, G. \& Wilson, D. (2010). Epistemic vigilance. Mind \& Language, 25(4), 359-393.

Swire, B., Berinsky, A. J., Lewandowsky, S. \& Ecker, U. K. (2017). Processing political misinformation: Comprehending the trump phenomenon. Royal Society open science, 4(3), 160802 . 
Swire, B., Ecker, U. K. \& Lewandowsky, S. (2017). The role of familiarity in correcting inaccurate information. Journal of experimental psychology: learning, memory, and cognition, 43(12), 1948.

Walter, N. \& Murphy, S. T. (2018). How to unring the bell: A meta-analytic approach to correction of misinformation. Communication Monographs, 85 (3), 423-441.

Walter, N. \& Tukachinsky, R. (2020). A meta-analytic examination of the continued influence of misinformation in the face of correction: How powerful is it, why does it happen, and how to stop it? Communication Research, 47(2), 155-177.

Westfall, J., Judd, C. M. \& Kenny, D. A. (2015). Replicating studies in which samples of participants respond to samples of stimuli. Perspectives on Psychological Science, 10(3), 390-399.

Wilkes, A. \& Leatherbarrow, M. (1988). Editing episodic memory following the identification of error. The Quarterly Journal of Experimental Psychology, 40(2), $361-387$. 